\title{
An Augmented PID Control Scheme for Robust Control
}

\author{
Mehmet Önder Efe \\ Hacettepe University \\ Dept. of Computer Engineering \\ Ankara, Turkey \\ onderefe@hacettepe.edu.tr
}

\begin{abstract}
This paper considers an augmentation of PID controller. The typical design approach for feedback control is to consider the nominal plant model and implement a PID controller. Yet the model mismatch is a significant issue that requires further tuning of PID parameters. This paper augments the PID controller with a fourth term to alleviate the difficulties caused by plant model mismatch. Using the proposed approach, practitioners follow the standard procedure for nominal plant and augment the control input with the proposed term with the real system. The tracking performances are discussed comparatively and results are found promising.
\end{abstract}

Keywords—sliding mode control; plan-model mismatch; robust control, augmented PID control

\section{INTRODUCTION}

Proportional Integral and Derivative (PID) type control has been the most influential control scheme used in a wide range of processes. The simple structure, simple explanation of the overall function and the algorithms to tune its parameters to observe certain types of responses make it preferable from a manufacturer's point of view. Availability of many patents for parameter tuning of PID controllers and the ongoing research on developing the variants of this scheme are the indicators of the popularity of PID control scheme. This has been the motivation of the current paper.

The design process for a PID controller is a well defined issue when the system under control is linear. Imposing the design constraints would yield the three gains to construct the PID controller. Today, such facilities are automated so that CAD software can perform the optimization as long as the feedback loop is closed and the peripheral devices are placed in the loop. Matlab/Simulink is one such example providing this kind of a tool.

Although there are motivating facts for using PID control, if one or few of the assumptions are removed, the performance of a feedback loop with a PID control can undergo sever degradations that require either complex parameter tuning algorithms or control schemes other than PID. It is well known that in both avenues there are many alternatives at various prices, i.e. computational complexity, hardware requirements, design needs, mathematical tractability etc. Having this picture in the front, one's attitude would be to enhance PID control scheme, if possible.
Toward this goal, in the literature several works need mentioning. The theory of PID control and basics of its industrial use is explained in [1], in which Aström and Hagglund emphasize the tuning issues. In [2], tuning issues are elaborated and in [3], a collection of articles from the field have been presented as a book. A closer look to the literature reveals that the popularity of PID control is ongoing in every direction. Sliding Mode Control (SMC), on the other hand, is another popular robust control scheme displaying several prominent features when there are uncertainties. The two phase responses of the system under control are the reaching phase and the sliding regime. State of the controlled system is guided toward a particular subspace where the trajectories within which end up with the origin. In the literature, numerous publications have been reported during the last few decades and the theory have reached a particular level of maturity. It is not surprising to see the blending of techniques to benefit from the good characteristics of every individual approach. SMC and PID collaboration is one such approach, [4-8].

In [4], control of a chaotic system is considered. PID controller is chosen to be an adaptive one and gradient method is chosen as the adaptation law. Supervisory control is postulated similar to what is presented here but our work differs from [4] in that our design is based on the nominal model and the errors in between nominal model and the true system. Further, despite the similarity in spirit, the structures of the control systems are fundamentally different. In [5], generalized PI control is blended with SMC and DC/DC boost and buck converters are considered for illustrating the proficiency of the approach. In [6], output tracking based discrete PID-SMC for multi input multi output systems is studied. The bang-bang nature of the conventional SMC scheme is criticized and the switching component of the control signal is produced by a PID controller admitting the switching variable as its input. The paper derives the necessary conditions for closed loop stability and performance. Another interesting combination is reported in [7], where the problem of slosh control is considered. Sliding mode observer and a SMC pair is used to generate the desired trajectory and a PID controller is used to control the permanent magnet DC motor. In [8], a mixture of PID and SMC schemes is presented to obtain a PID based sliding surface and a successful application is demonstrated on a 2 dof robotic system. 
It is possible to increase the number of references considering such successful PID plus SMC integration but due to the space limit interested reader is referred to the listed references in the cited works. Another ingredient to SMC and PID combination is the intelligence and adaptive structures, such as fuzzy logic, neural networks or support vector machines. A good survey on this is provided in [9] and the references therein.

This paper is organized as follows: The second section introduces the assumptions and gives the problem definition. The claim and stability proof is provided. The third section discusses two sets of simulations. The last part of the paper gives the concluding remarks.

\section{PROBLEM DEFINITON}

\section{A. Conventional Approach}

Let the system in (1)-(2) is the nominal model of the system under control. A second order process is chosen to demonstrate the idea yet the approach to be discussed is applicable to higher order process models where we can design PID control mechanisms.

$$
\begin{gathered}
{\left[\begin{array}{l}
\dot{x}_{1} \\
\dot{x}_{2}
\end{array}\right]=\left[\begin{array}{cc}
0 & 1 \\
-a & -b
\end{array}\right]\left[\begin{array}{l}
x_{1} \\
x_{2}
\end{array}\right]+\left[\begin{array}{l}
0 \\
c
\end{array}\right] u} \\
y_{n}=x_{1}
\end{gathered}
$$

With zero initial conditions, the nominal system has the transfer function given in (3), where we assume $c>0$, and $a$, $b \neq 0$ and the poles of the nominal model are stable. The goal of the control system design is to force $e_{1}:=r_{1}-x_{1} \rightarrow 0$, $e_{2}:=r_{2}-x_{2} \rightarrow 0$ with $\dot{r}_{1}=r_{2}$ are the reference signals for position and velocity, $e_{1}$ and $e_{2}$ are the position and velocity tracking errors, respectively.

$$
G_{n}(s)=\frac{c}{s^{2}+b s+a}
$$

Considering this system in the classical unity negative feedback loop, imposed transient and steady state conditions will let us compute the PID controller gains as given below.

$$
U_{P I D}(s)=\left(k_{p}+k_{i} / s+k_{d} s\right) E(s)
$$

where $E(s)$ is the Laplace transform of $e_{1}(t)$. For the rest of the paper, our assumption is the stability of nominal system in (1)(2) and the fixed coefficient PID controller in (4), and this pair meets the desired performance specifications when $u=u_{P I D}$.

\section{B. Proposed Approach}

Consider the block diagram in Fig. 1. Since the PID controller has been designed according to the information put forth by the nominal model, the bottom loop forces $e_{1} \rightarrow 0$.

With $\lambda>0$ being the slope parameter and $M>0$ being the magnitude parameter and $e_{m}:=y_{n}-y_{p}$ being the model following error, we choose

$$
\begin{gathered}
\sigma:=\dot{e}_{m}+\lambda e_{m} \\
u_{S M C}:=M \operatorname{sgn}(\sigma)
\end{gathered}
$$

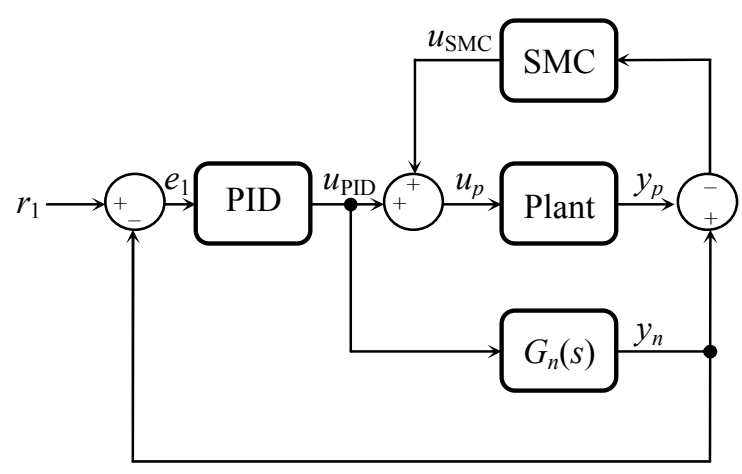

Fig. 1. Block diagram of the proposed control system

The interpretation of the above selection is as follows: The nominal system forces the reference trajectory and the uncertain plant is forced to follow the nominal system. The above choice makes $\mathrm{s}=0$ an attractor and once the error gets trapped to this subspace, it lies on it forever. Eventually the model following error will exhibit $e_{m} \rightarrow 0$ as time passes. Below theorem states the necessary conditions for closed loop stability of the overall system.

Theorem: Let (7)-(8) be the system under control and let (1)-(2) be a linearized model of it. Let the uncertainties satisfy $\left|D_{1}\left(x_{1 \mathrm{p}}, x_{2 \mathrm{p}}\right)\right|<B_{f}$ and $0<\left|D_{2}\left(x_{1 \mathrm{p}}, x_{2 \mathrm{p}}\right)\right|<B_{g}<c$.

$$
\begin{gathered}
{\left[\begin{array}{c}
\dot{x}_{1 p} \\
\dot{x}_{2 p}
\end{array}\right]=\left[\begin{array}{cc}
0 & 1 \\
-a & -b
\end{array}\right]\left[\begin{array}{l}
x_{1 p} \\
x_{2 p}
\end{array}\right]+\left[\begin{array}{c}
0 \\
D_{1}(., .)
\end{array}\right]+\left[\begin{array}{c}
0 \\
c+D_{2}(., .)
\end{array}\right] u} \\
y_{p}=x_{1 p}
\end{gathered}
$$

Let the nominal system in (1)-(2) and the PID controller in (4) be a stable pair in negative feedback loop and for a Lyapunov function $V_{1}\left(e_{1}, \mathrm{~d} e_{1} / \mathrm{d} t\right)$, the negativity of the time derivative of $V_{1}$ is ensured by the fixed PID controller. Then, for every $\varepsilon>0$, choosing

$$
M=(1+\varepsilon)\left\{\frac{\left|a e_{m}+(b+\lambda) \dot{e}_{m}\right|+B_{g}\left|u_{P I D}\right|+B_{f}}{c-B_{g}}\right\}
$$

and

$$
u_{p}:=u_{P I D}+u_{S M C}
$$

makes $\sigma=0$ a stable attractor and the uncertain system in (7)(8) is forced to enter sliding regime.

Proof: Let the control Lyapunov function (clf) be chosen as

$$
V=V_{1}\left(e_{1}, \dot{e}_{1}\right)+\frac{1}{2} \sigma^{2}
$$


Taking the time derivative leads to

$$
\dot{V}=\dot{V}_{1}\left(e_{1}, \dot{e}_{1}\right)+\sigma \dot{\sigma}
$$

According to (5)

$$
\dot{\sigma}:=\ddot{e}_{m}+\lambda \dot{e}_{m}
$$

Utilizing the nominal system dynamics and the uncertain system dynamics, we have

$$
\begin{aligned}
\dot{\sigma}:= & \left(-a e_{m}+(\lambda-b) \dot{e}_{m}-D_{1}-D_{2} u_{P I D}\right) \\
& -\left(c+D_{2}\right) M \operatorname{sgn}(\sigma)
\end{aligned}
$$

In order to have a negative time derivative for the clf in (11), we need to have $\dot{\sigma} \sigma:<0$. Multiplying both sides of (14) with $\sigma$ yields

$$
\begin{aligned}
\dot{\sigma} \sigma & :=\left(-a e_{m}+(\lambda-b) \dot{e}_{m}-D_{1}-D_{2} u_{P I D}\right) \sigma \\
& -\left(c+D_{2}\right) M|\sigma| \\
& <\left|-a e_{m}+(\lambda-b) \dot{e}_{m}-D_{1}-D_{2} u_{P I D}\right||\sigma| \\
& -\left(c-\left|D_{2}\right|\right) M|\sigma| \\
& \leq\left(\left|-a e_{m}+(\lambda-b) \dot{e}_{m}\right|+\left|D_{1}\right|+\left|D_{2}\right|\left|u_{P I D}\right|\right)|\sigma| \\
& -\left(c-\left|D_{2}\right|\right) M|\sigma| \\
& <\left(\left|-a e_{m}+(\lambda-b) \dot{e}_{m}\right|+B_{f}+B_{g}\left|u_{P I D}\right|\right)|\sigma| \\
& -\left(c-\left|D_{2}\right|\right) M|\sigma|
\end{aligned}
$$

For $\dot{\sigma} \sigma:<0$, the above inequality imposes

$$
M>\frac{\left|-a e_{m}+(\lambda-b) \dot{e}_{m}\right|+B_{f}+B_{g}\left|u_{P I D}\right|}{c-B_{g}}
$$

This result justifies the choice in (9) and ensures $\dot{V}=\dot{V}_{1}\left(e_{1}, \dot{e}_{1}\right)+\sigma \dot{\sigma}<0$.

\section{SimUlations}

Two sets of simulations have been conducted. In the first set the reference signal is $r_{1}(t)=\sin (0.8 t)$, which is continuously differentiable. The second set of simulations consider a discontinuous reference signal given by $r_{1}(t)=\operatorname{sign}(\sin (0.8 t))$. The other parameters in both simulations have been kept the same and the values are as follows: $a=-4, b=-3, c=20$. For this nominal system, we set $k_{p}=5.55, k_{i}=6.40, k_{d}=1.17$ with filter coefficient for derivative computation is 87.5, slope constant $\lambda=5$. Sign function is approximated as $\operatorname{sign}(\sigma) \cong \sigma$ $/(|\sigma|+\eta)$ with $\eta=0.1$, and $\varepsilon$ of (10) is chosen as $\varepsilon=10$.

We studied the following bounded uncertainties for the plant under control.

$$
D_{1}=\frac{4 \sin \left(x_{1 p}\right) \cos \left(x_{2 p}\right)}{1+x_{2 p}^{2}}<4=B_{f}
$$

$$
D_{2}=0.1 \frac{1+\sin \left(x_{1 p x_{2 p}}\right)}{1+x_{2 p}^{2}}<0.2=B_{g}
$$

In the simulations, the control signals applied to the nominal model and the full model are saturated at \pm 10 . This is a significant difficulty as the control law with the introduced discontinuous term is supposed to produce large magnitude control signals yet the physical constraints will limit these signals to applicable levels. It is our understanding that obtaining good tracking performance under such a restriction would be valuable for practicing engineers.

Results of the first experiment are shown in Figs. 2-4. Fig. 2 shows the responses without the proposed scheme. The reference signal is changing and the nominal system response quickly starts following the reference signal as expected, whereas, the response of the plant $\left(y_{p}\right)$ does not display a satisfactory performance. This figure dhows that the designer should either change his/her approach or to continue using nominal model with a modification to the feedback control law. Fig. 3 has four subplots. Top left depicts the outputs together. After a very quick transient regime, the control law in (10) drives the system toward the response of the nominal model. Top right subplot of Fig. 3 illustrates the applied control signal, bottom left illustrates the time evolution of SMC gain $(M)$ and the bottom right subplot depicts the phase plane spanned by the nominal model following error and its time derivative. The path followed is quite promising as it is attracted by the loci defined by $\sigma=0$ and is slides down to origin according to (5). As we set $\lambda=5$, the slope of the line followed in the phase space is $-\lambda$.

The signals shown in Fig. 4 are the same as those in Fig. 3 but Fig. 4 zooms for the initial behaviors. The signals produced during the reaching phase are smooth enough to be generated by an ordinary computing facility.

The second experiment considers the discontinuous reference signals. When the proposed augmentation is turned off, the responses are shown in Fig. 5, where we see that the nominal model performs well whereas the plant containing uncertainties degrade the tracking performance significantly.

In Fig. 6, the responses with the discontinuous reference signal are shown with the proposed SMC term. The adverse effects of the uncertainties are removed perfectly and the full system follows the reference signal precisely. To see the reaching phase, the initial values of the outputs are chosen as nonzero and apart from each other and the closed loop system exhibits the desired characteristics of the sliding regime.

Similarly, the responses are zoomed in Fig. 7 to unveil the details of what happened during the early phase of the simulation. The control signal rings when $t \in(0.09,0.14) \mathrm{sec}$. Further zooming stipulates that the signal produced can be generated by ordinary microprocessor hardware.

\section{CONCLUSIONS}

This paper considers an augmented PID control approach. Considering the widespread use of PID control and continuous efforts to improve its performance and increasing number of 
filed patents on the topic motivate us study the PID control scheme to observe sliding motion and exhibit the desired characteristics of sliding mode control technique.

In order to achieve the goal, the PID scheme is extended with a discontinuous term. The argument of this term is a switching variable, which receives its subcomponents from a nominal model and the full model. The scheme schedules a gain, which is denoted by $M$ in the paper, and several design parameters are chosen by the designer.

The paper considers two exemplar cases with continuous reference signal and with a discontinuous reference signal. In both cases, it is observed that the designed PID controller using only the nominal model is incapable of producing satisfactory results. The proposed method significantly improves the tracking performance without introducing fast changes to the control signal.

\section{ACKNOWLEDGEMENTS}

The author gratefully acknowledges the facilities of the Computer Engineering Department of Hacettepe University and its library.

\section{REFERENCES}

[1] K.J. Astrom, T Hägglund, PID Controllers: Theory, Design and Tuning, ISA, 1995.

[2] K. Papadopoulos, PID Controller Tuning Using the Magnitude Optimum Criterion, Springer, 2014.

[3] R. Vilanova, A. Visioli, (Eds.), PID Control in the Third Millennium: Lessons Learned and New Approaches, Springer 2014.

[4] W.-D. Chang, J.-J. Yan, "Adaptive Robust PID Controller Design Based on a Sliding Mode for Uncertain Chaotic Systems," Chaos Solitons \& Fractals, v.26, pp.167-175, 2005.

[5] H. Sira-Ramirez, "On the generalized PI sliding mode control of DC-toDC power converters: A tutorial," Int.J. Control, v.76, pp.1018-1033, 2003.

[6] Y. Cao, X.B. Chen, "An Output-Tracking-Based Discrete PID-Sliding Mode Control for MIMO Systems," IEEE/ASME Trans. on Mechatronics, v.19, no.4, pp.1183-1194, 2014.

[7] B. Bandyopadhyay, P.S. Gandhi, S. Kurode, "Sliding Mode Observer Based Sliding Mode Controller for Slosh-Free Motion Through PID Scheme," IEEE Trans. on Industrial Electronics, vol.56, no.9, pp.34323442, Sep. 2009.

[8] E.M. Jafarov, M.N.A. Parlakçı, Y. İstefanopulos, "A New Variable Structure PID-Controller Design for Robot Manipulators," IEEE Trans. on Control Systems Technology, v.13, no.1, pp.122-130, Jan. 2005.

[9] O. Kaynak, K. Erbatur and M. Ertugrul, "The Fusion of Computationally Intelligent Methodologies and Sliding-Mode Control—A Survey," IEEE Trans. on Industrial Electronics, v.48, no.1, pp.4-17, Feb. 2001

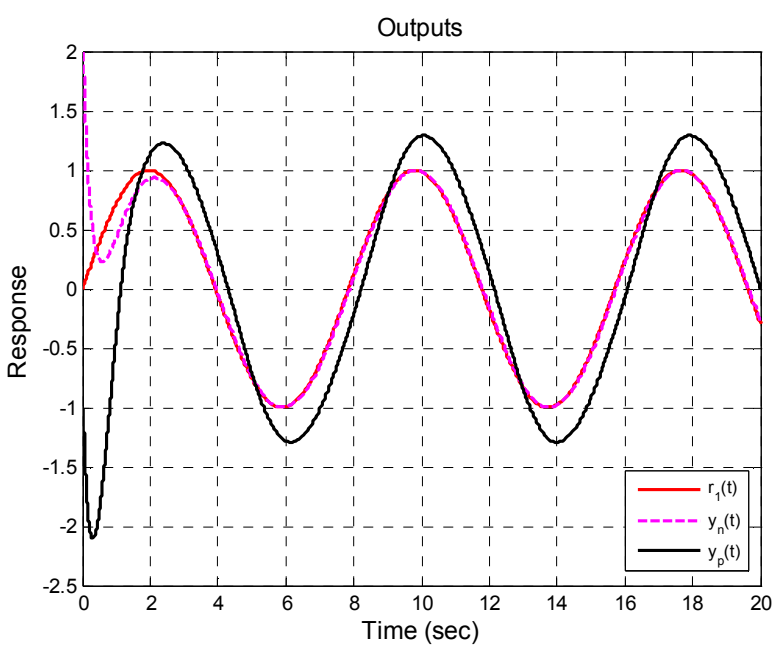

Fig. 2. Responses without SMC term for continuous reference signal
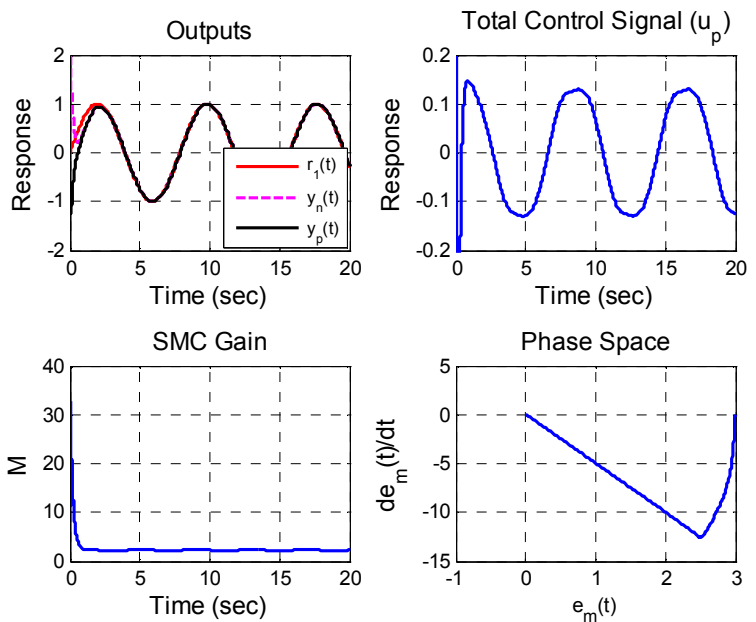

Fig. 3. Outputs, control signal, SMC gain $(M)$ and phase space behavior for continuous reference signal
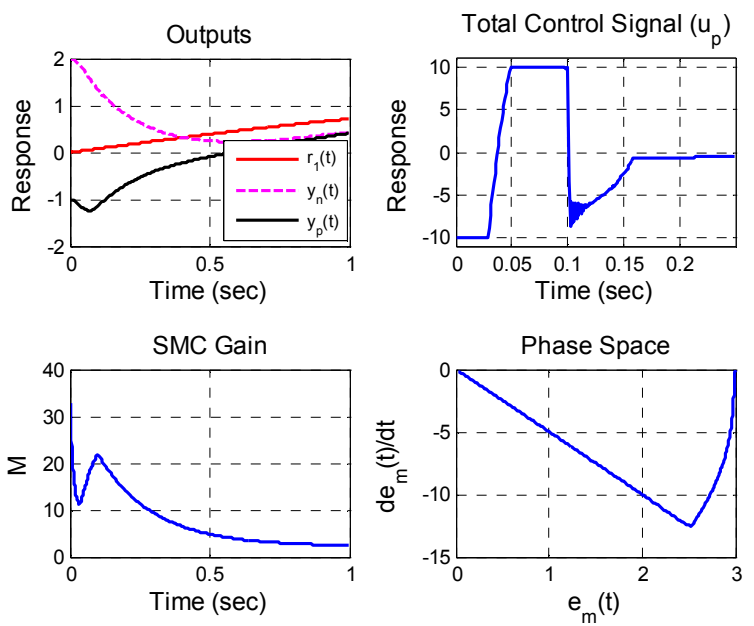

Fig. 4. Outputs, control signal, SMC gain $(M)$ and phase space behavior for continuous reference signal (Transient regime) 


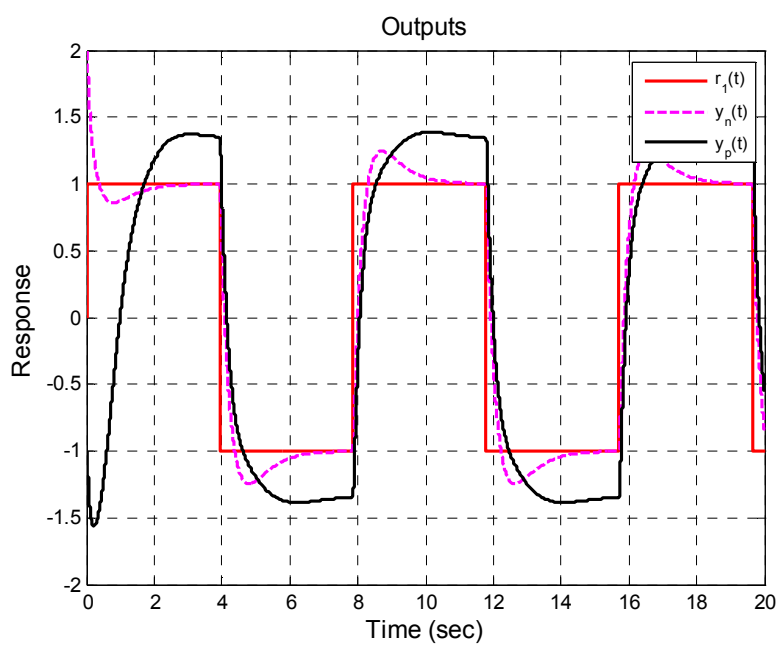

Fig. 5. Responses without SMC term for discontinuous reference signal
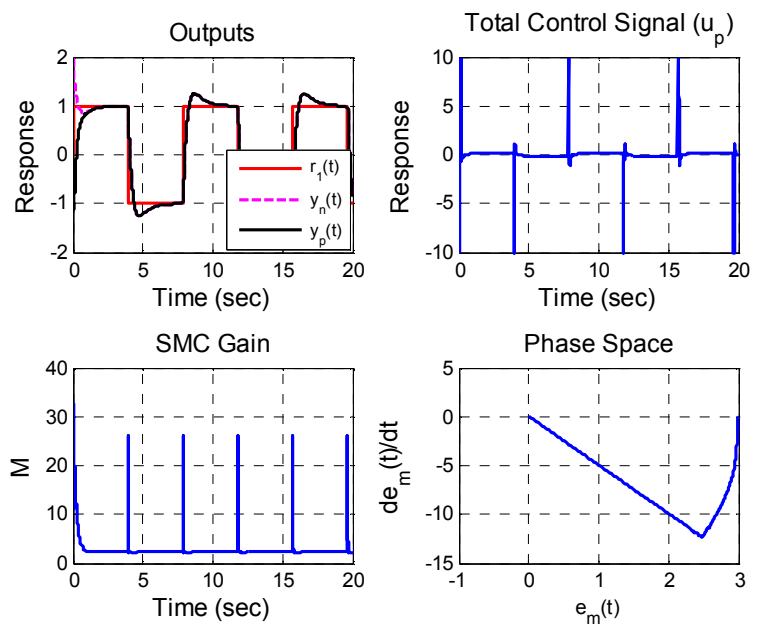

Fig. 6. Outputs, control signal, SMC gain $(M)$ and phase space behavior for discontinuous reference signal
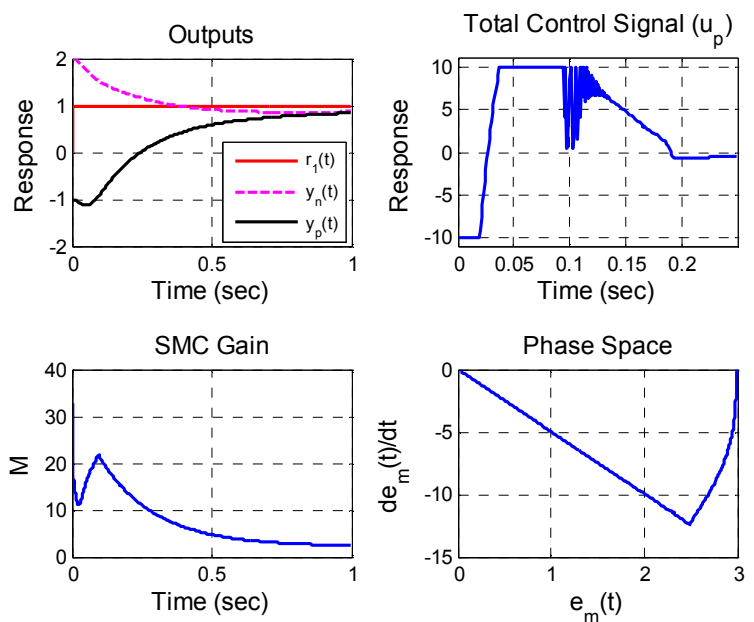

Fig. 7. Outputs, control signal, SMC gain $(M)$ and phase space behavior for discontinuous reference signal (Transient regime) 